
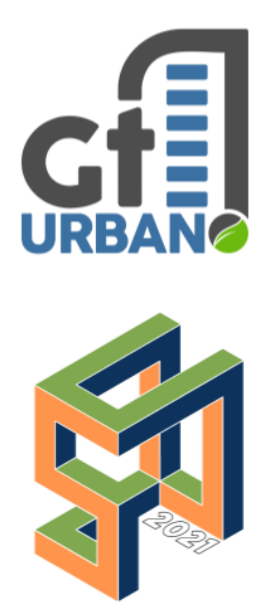

SINGEURB

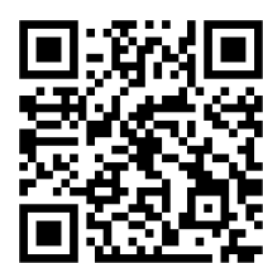

Como citar:

BECKER, Débora; REIS, Antônio Tarcísio. Intensidade de uso diurno dos espaços abertos públicos adjacentes a condomínios fechados e a edificações tradicionais. In: III SIMPÓSIO NACIONAL DE GESTÃO E ENGENHARIA URBANA: SINGEURB, 2021, Maceió. Anais... Porto Alegre: ANTAC, 2021. p. 1-9. Disponível em: https://eventos.antac. org.br/index.php/sin geurb/issue/view/14

\title{
Intensidade de uso diurno dos espaços abertos públicos adjacentes a condomínios fechados e a edificações tradicionais
}

Intensity of day time use of public open spaces adjacent to gated communities and traditional buildings

Débora Becker, Universidade Federal do Rio Grande do Sul (UFRGS), debora.becker@ufrgs.br Antônio Tarcísio Reis, Universidade Federal do Rio Grande do Sul (UFRGS), tarcisio@orion.ufrgs.br

\section{RESUMO}

Os condomínios fechados estão transformando a paisagem urbana contemporânea, com a substituição das fachadas das edificações tradicionais, com portas e janelas voltadas para a rua, por suas barreiras físicas contínuas. O objetivo deste trabalho é identificar a intensidade de uso diurno nos espaços abertos públicos adjacentes a condomínios fechados e a edificações tradicionais, e as relações com as conexões funcionais e visuais, a quantidade de sacadas e as barreiras físicas das edificações. É parte de uma pesquisa de doutorado que investiga o impacto dos condomínios fechados no espaço urbano. Quinze quadras, localizadas na cidade de Porto Alegre/RS, foram selecionadas e classificadas em cinco tipos, de acordo com a combinação das seguintes interfaces: muros de condomínios fechados; predomínio de edificações tradicionais; e praças. Os meios de coleta de dados incluem: levantamento físico e observações de comportamento, dados quantitativos espacializados em ambiente SIG e analisados através de testes estatísticos. Os resultados evidenciam os efeitos negativos das barreiras físicas contínuas dos condomínios fechados sobre a intensidade de uso diurno dos espaços abertos públicos e reforçam a importância da permeabilidade física das edificações para a vitalidade urbana de áreas residenciais.

Palavras-chave: Uso dos espaços abertos públicos, Conexões funcionais e visuais, Barreiras físicas, Condomínios fechados.

\section{ABSTRACT \\ Gated communities are transforming the contemporary urban landscape, replacing the facades of traditional buildings, with doors and windows facing the street, for their continuous physical barriers. The objective of this work is to identify the intensity of daytime use in public open spaces adjacent to closed condominiums and traditional buildings, and the relationships with functional and visual connections, the number of balconies and physical barriers of the buildings. It is part of a doctoral research that investigates the impact of closed condominiums on urban space. Fifteen blocks, located in the city of Porto Alegre/RS, were selected and classified into five types, according to the combination of the following interfaces:}


walls of closed condominiums; predominance of traditional buildings; and squares. The means of data collection include: physical survey and behavioral observations, quantitative data spatialized in a GIS environment and analyzed through statistical tests. The results show the negative effects of the continuous physical barriers of closed condominiums on the intensity of daytime use of public open spaces and highlight the importance of the physical permeability of buildings for the urban vitality of residential areas.

Keywords: Use of public open spaces, Functional and visual connections, Physical barriers, Gated communitie.

\section{INTRODUÇÃO}

A importância da relação entre os espaços abertos públicos e os edificados para a vitalidade urbana tem sido destacada por vários autores nas últimas décadas, a exemplo de Jan Gehl (1987, 2010) em seus livros "Life Between Building: Using Public Space" e "Cities for People". Nesse sentido, a quantidade de conexões funcionais das edificações (acessos a pedestres) afeta diretamente a intensidade de uso nos espaços abertos públicos: quanto maior seu número, maior o potencial de movimento (HILLIER e HANSON, 1984; ANTOCHEVIZ et al., 2019). Por sua vez, as conexões visuais (janelas, vitrines e portas transparentes) possibilitam a supervisão dos espaços abertos públicos e tornam a experiência urbana mais estimulante, atraindo e gerando possibilidades de encontros (BENTLEY et al, 1985; JACOBS, 2000; GHEL 2010). Particularmente para as áreas residenciais, os recuos de jardins e as sacadas das edificações, assumem especial importância para animação dos espaços abertos públicos (HERTZERGER, 1999) a partir da realização tanto de atividades cotidianas quanto de lazer e interação social (BECKER, 2005; GEHL, et. al., 2006).

Por outro lado, os condomínios horizontais murados têm alterado as características espaciais e morfológicas das cidades (WEBSTER et al., 2002; BORSDORF, 2003), criando barreiras funcionais e visuais contínuas, em oposição às fachadas tradicionais, com portas e janelas voltados para a rua, (REIS e BECKER, 2011). Entretanto, esses condomínios têm sido construídos em larga escala em distintas cidades de diferentes países, incluindo o Brasil (GRANT e MITTELSTEADT, 2004; ESTEVES e NOGUEIRA, 2013). Ainda, tende a haver um maior efeito dos diferentes níveis de conexões visuais e funcionais sobre o uso diurno dos espaços abertos públicos, do que sobre o uso noturno, quando existe uma redução na quantidade de conexões ativas. Logo, embora existam estudos que evidenciam o impacto negativo dos condomínios fechados no uso dos espaços urbanos (BECKER 2005), ainda é necessário produzir e disseminar mais evidências, além de aprofundar o conhecimento existente. Assim, é objetivo deste identificar a intensidade de uso diurno nos espaços abertos públicos adjacentes a condomínios fechados e a edificações tradicionais, incluindo os pátios frontais visíveis, e as relações com as conexões funcionais, as conexões visuais, a quantidade de sacadas e as barreiras físicas das edificações.

\section{METODOLOGIA}

Este estudo foi realizado em Porto Alegre/RS, que retrata a expansão dos condomínios fechados $(\mathrm{KOCH}$, 2008; MAMMARELLA e BARCELOS, 2008; UEDA, 2005). A partir de um mapeamento dos principais condomínios fechados de alta renda foram selecionados sete localizados em zona de tecido urbano consolidado (bairro Três Figueiras), circundados por barreira física e visual contínua com mais de cem metros de extensão em pelo menos uma face, construídos há mais de um ano e próximos de vias delimitadas em ambos os lados por edificações tradicionais (Figura 1). 
Figura 1 - Condomínios fechados selecionados - Bairro Três Figueiras

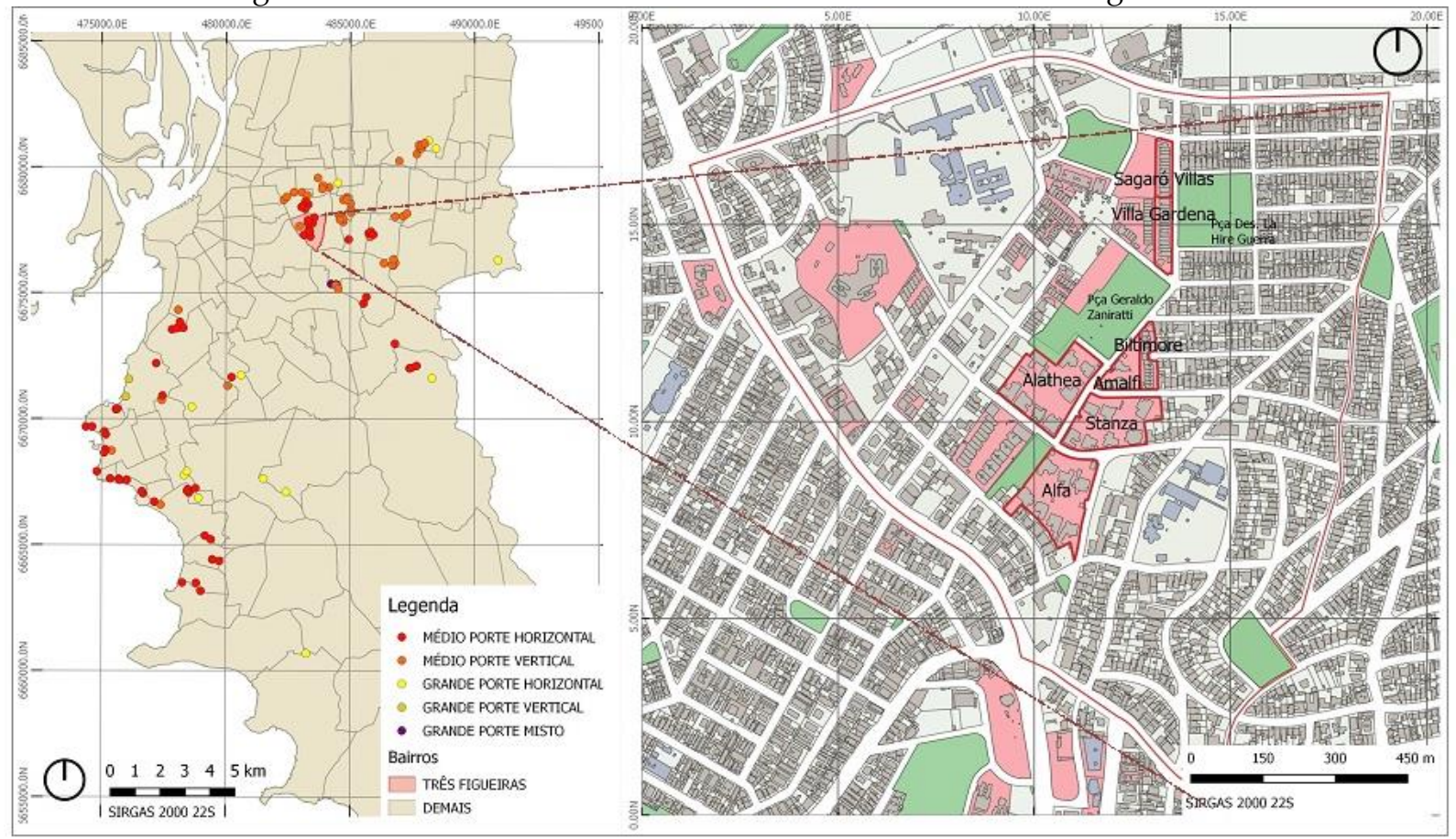

Fonte: Mapa elaborado pelos autores - software QGIS (2020)

No entorno desses sete condomínios fechados, foram selecionadas 15 quadras, com uso predominantemente residencial unifamiliar e distantes até 03 passos topológicos de vias principais (coletoras ou arteriais), categorizadas em cinco tipos de acordo com as características de suas interfaces (Figuras 2 e 3):

- $\quad$ Tipo 1 - predomínio de edificações tradicionais, com portas e janelas voltadas para as ruas, em ambos os lados da quadra;

- $\quad$ Tipo 2 - edificações tradicionais em um lado da quadra e praça de outro;

- $\quad$ Tipo 3 - edificações tradicionais em um lado da quadra e muro de condomínio fechado de outro;

- $\quad$ Tipo 4 - praça de um lado da quadra e muro de condomínio fechado de outro;

- $\quad$ Tipo 5 - muros de condomínios fechados em ambos os lados da quadra;

Além do levantamento de arquivo, com dados obtidos junto à Prefeitura Municipal de Porto Alegre e imagens de satélite, foram realizados levantamentos físicos detalhados e observações de comportamento nas 15 quadras investigadas.

Figura 2 - Quadras selecionadas e Percurso 1 


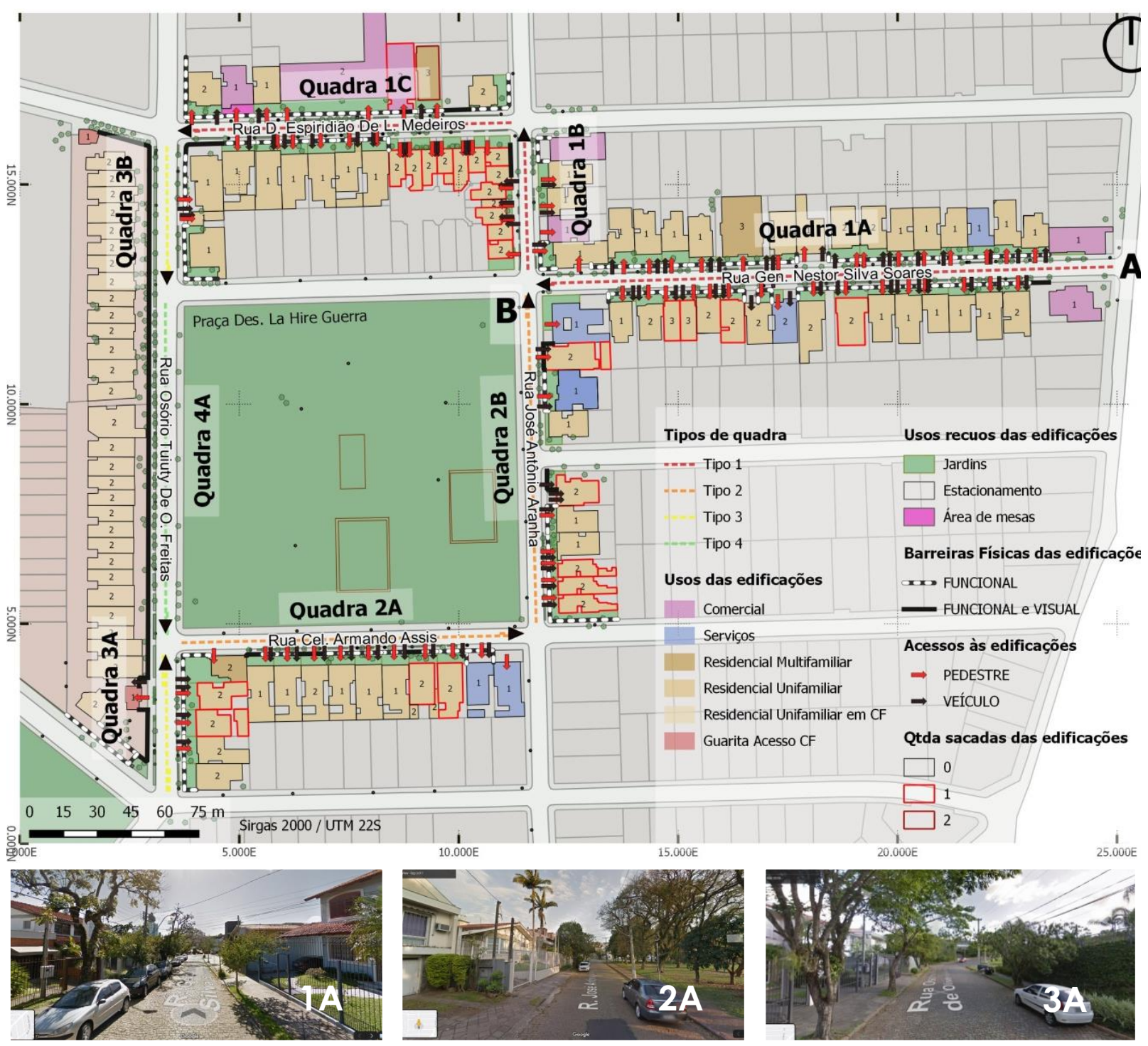

Fonte: Mapa elaborado pelos autores - software QGIS. Imagens street view (2020)

As observações de comportamento foram realizadas em seis dias de semana e dois finais de semana, nos períodos da manhã e tarde (totalizando 20 turnos), nos horários entre $09 \mathrm{hs}$ e $11 \mathrm{~h} 30 \mathrm{~min}$, e entre $14 \mathrm{hs}$ e 16h30min, durante os meses de dezembro de 2019, janeiro e março de 2020. Foram definidos dois percursos, o primeiro no entorno dos condomínios Sagaró Vilas e Villa Gardena, localizados a norte (Figura 2), e o segundo no entorno dos condomínios Baltimore, Alathea, Stanza, Amalfa e Alfa (Figura 3), localizados a sul. 
Figura 3 - Quadras selecionadas - Percurso 2

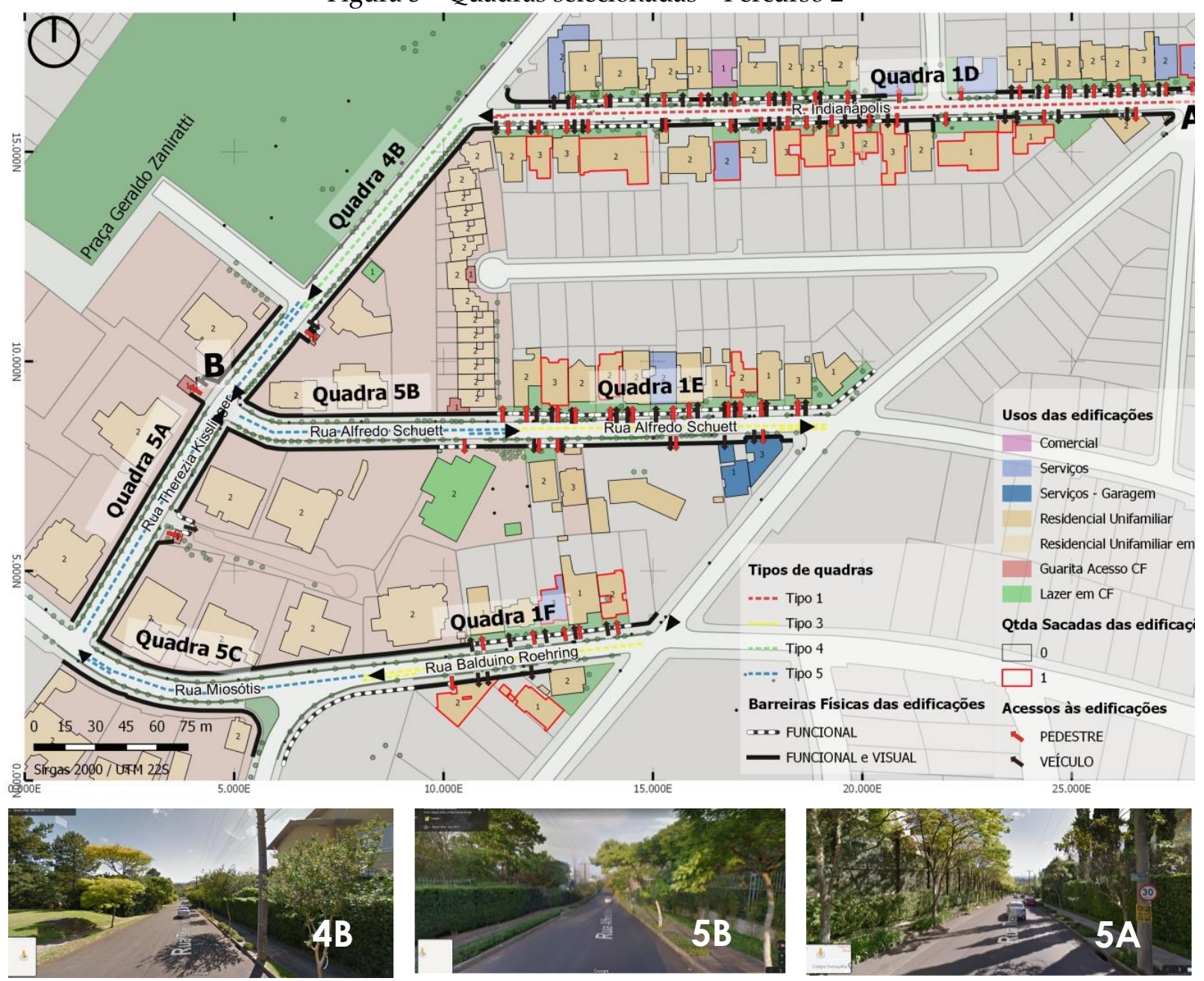

Fonte: Mapa elaborado pelos autores - software QGIS. Imagens street view (2020)

Os dados obtidos foram espacializados e quantificados em ambiente SIG, com uso do software Quantum Gis, versão 2.18.2, para possibilitar a verificação das relações entre: conexão funcional (número de acessos a pedestre); conexão visual (comprimento linear em metro das transparências); barreiras físicas e visuais (comprimento linear em metro); e supervisão por sacada (número de sacadas por edificação); e usos existentes. Essas medidas foram transformadas em taxas por meio da razão entre a soma das medidas de cada variável nos dois lados da quadra e a soma do comprimento de cada lado da quadra, e multiplicado por 100 para representar a quantificação de cada variável em 100 metros. Tais dados foram analisados através do teste de correlação Pearson no programa estatístico SPSS27 (Statical Package for the Social Sciences).

\section{RESULTADOS}

Considerando a taxa geral de uso diurno (seis dias da semana e os dois finais de semana) nas 15 quadras (Gráfico 1), as duas quadras tipo 2 (2A e 2B - edificações tradicionais em um lado da quadra e praça no outro) são as que, claramente, possuem as maiores taxas (5,59 e 3,68, respectivamente). Por outro lado, as três quadras tipo 5 (5A, 5B e 5C - muros de condomínios fechados em ambos os lados) são as que possuem as taxas mais baixas $(0,54,0,21$ e 0,17 respectivamente), expressivamente menores (entre quase sete até trinta 
e três vezes mais baixas) que aquelas nas quadras tipo 2. As seis quadras tipo 1 (edificações tradicionais em ambos os lados) apresentam dois padrões: enquanto as quadras 1A, 1B e 1C, possuem as segundas maiores taxas $(2,08,2,02,2,76$, respectivamente), as quadras $1 \mathrm{D}, 1 \mathrm{E}$ e $1 \mathrm{~F}$ possuem taxas visivelmente menores $(1,19$, 1,37 e 1,63, respectivamente), embora bem maiores do que aquelas nas quadras tipo 5 . As duas quadras tipo 3 (edificações tradicionais em um lado e muros de condomínios fechados no outro), bem como as duas quadras tipo 4 (muros de condomínios fechados de um lado e praça de outro) também apresentam padrões diferentes: enquanto a quadra $3 \mathrm{~A}$ possui taxa de 2,03 , as quadras $3 \mathrm{~B}$ e $4 \mathrm{~A}$ possuem taxas bem mais baixas $(1,12$ e 1,11, respectivamente), embora claramente superiores à taxa na quadra $4 \mathrm{~B}(0,40)$.

Gráfico 1 - Taxas de uso diurno de cada quadra

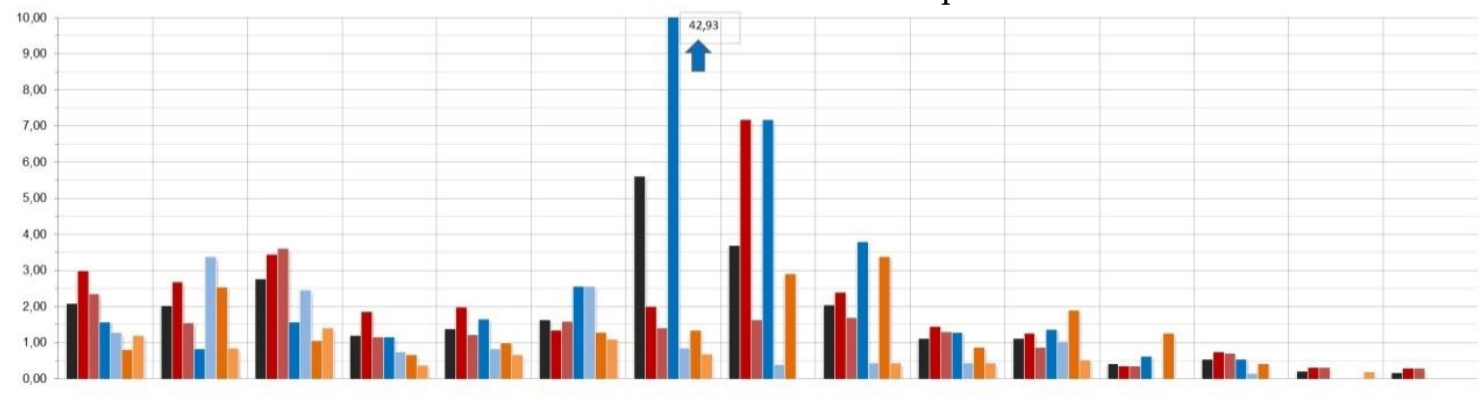

\begin{tabular}{|c|c|c|c|c|c|c|c|c|c|c|c|c|c|c|c|c|}
\hline & Tipo & 1 & 1 & 1 & 1 & 1 & 1 & 2 & 2 & 3 & 3 & 4 & 4 & 5 & 5 & 5 \\
\hline & Quadra & A & B & $\mathrm{C}$ & D & $\mathbf{E}$ & $\mathbf{F}$ & A & B & A & B & A & B & A & B & C \\
\hline \multicolumn{2}{|c|}{ Média Geral } & $\begin{array}{l}2,08 \\
(211)\end{array}$ & $\begin{array}{c}2,02 \\
(48)\end{array}$ & $\begin{array}{l}2,76 \\
(158)\end{array}$ & $\begin{array}{l}1,19 \\
(163)\end{array}$ & $\begin{array}{l}1,37 \\
(83)\end{array}$ & $\begin{array}{l}1,63 \\
(89)\end{array}$ & $\begin{array}{l}5,59 \\
(336)\end{array}$ & $\begin{array}{l}3,68 \\
(190)\end{array}$ & $\begin{array}{c}2,03 \\
(48)\end{array}$ & $\begin{array}{l}1,12 \\
(26)\end{array}$ & $\begin{array}{l}1,11 \\
(65)\end{array}$ & $\begin{array}{l}0,40 \\
(19)\end{array}$ & $\begin{array}{c}0,54 \\
(39)\end{array}$ & $\begin{array}{l}0,21 \\
\text { (11) }\end{array}$ & $\begin{array}{l}0,17 \\
(08)\end{array}$ \\
\hline \multirow{2}{*}{ 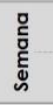 } & Manhã & $\begin{array}{c}2,99 \\
(91)\end{array}$ & $\begin{array}{c}2,66 \\
(19)\end{array}$ & $\begin{array}{c}3,43 \\
(59)\end{array}$ & $\begin{array}{l}1,85 \\
(76)\end{array}$ & $\begin{array}{l}1,98 \\
(36)\end{array}$ & $\begin{array}{l}1,34 \\
(22)\end{array}$ & $\begin{array}{c}2,00 \\
(36)\end{array}$ & $\begin{array}{l}7,17 \\
\text { (111) }\end{array}$ & $\begin{array}{c}2,40 \\
(17)\end{array}$ & $\begin{array}{c}1,43 \\
(10)\end{array}$ & $\begin{array}{l}1,26 \\
(22)\end{array}$ & $\begin{array}{c}0,35 \\
(05)\end{array}$ & $\begin{array}{c}0,73 \\
(16)\end{array}$ & $\begin{array}{l}0,31 \\
(05)\end{array}$ & $\begin{array}{c}0,28 \\
(04)\end{array}$ \\
\hline & Tarde & $\begin{array}{c}2,33 \\
(71)\end{array}$ & $\begin{array}{l}1,54 \\
\text { (11) }\end{array}$ & $\begin{array}{c}3,60 \\
(62)\end{array}$ & $\begin{array}{l}1,15 \\
(47)\end{array}$ & $\begin{array}{l}1,21 \\
(22)\end{array}$ & $\begin{array}{l}1,58 \\
(26)\end{array}$ & $\begin{array}{l}1,39 \\
(25)\end{array}$ & $\begin{array}{l}1,61 \\
(25)\end{array}$ & $\begin{array}{l}1,69 \\
(12)\end{array}$ & $\begin{array}{l}1,29 \\
(09)\end{array}$ & $\begin{array}{c}0,86 \\
(15)\end{array}$ & $\begin{array}{l}0,35 \\
(05)\end{array}$ & $\begin{array}{c}0,69 \\
(15)\end{array}$ & $\begin{array}{l}0,31 \\
(05)\end{array}$ & $\begin{array}{l}0,28 \\
(04)\end{array}$ \\
\hline \multirow{2}{*}{$\begin{array}{l}\text { 움 } \\
\text { 움 } \\
\text { 욤 }\end{array}$} & Manhã & $\begin{array}{l}1,58 \\
(16)\end{array}$ & $\begin{array}{l}0,84 \\
(02)\end{array}$ & $\begin{array}{l}1,57 \\
(09)\end{array}$ & $\begin{array}{l}1,17 \\
(16)\end{array}$ & $\begin{array}{l}1,65 \\
(10)\end{array}$ & $\begin{array}{c}2,56 \\
(14)\end{array}$ & $\begin{array}{c}42,93 \\
(258)\end{array}$ & $\begin{array}{l}7,17 \\
(37)\end{array}$ & $\begin{array}{l}3,80 \\
(09)\end{array}$ & $\begin{array}{l}1,29 \\
(03)\end{array}$ & $\begin{array}{l}1,37 \\
(08)\end{array}$ & $\begin{array}{l}0,63 \\
(03)\end{array}$ & $\begin{array}{l}0,55 \\
(04)\end{array}$ & $\begin{array}{c}0,00 \\
(0)\end{array}$ & $\begin{array}{c}0,00 \\
(0)\end{array}$ \\
\hline & Tarde & $\begin{array}{l}1,28 \\
(13)\end{array}$ & $\begin{array}{l}3,36 \\
(08)\end{array}$ & $\begin{array}{c}2,44 \\
(14)\end{array}$ & $\begin{array}{l}0,73 \\
(10)\end{array}$ & $\begin{array}{c}0,82 \\
(05)\end{array}$ & $\begin{array}{c}2,56 \\
(14)\end{array}$ & $\begin{array}{c}0,83 \\
(05)\end{array}$ & $\begin{array}{l}0,39 \\
(02)\end{array}$ & $\begin{array}{l}0,42 \\
(01)\end{array}$ & $\begin{array}{l}0,43 \\
(01)\end{array}$ & $\begin{array}{l}1,03 \\
(06)\end{array}$ & $\begin{array}{c}0,00 \\
(0)\end{array}$ & $\begin{array}{l}0,14 \\
(01)\end{array}$ & $\begin{array}{l}0,00 \\
(0)\end{array}$ & $\begin{array}{c}0,00 \\
(0)\end{array}$ \\
\hline \multirow{2}{*}{$\begin{array}{l}\text { 을 } \\
\frac{\mathrm{E}}{\mathrm{E}} \\
\text { 음 }\end{array}$} & Manhã & $\begin{array}{l}0,79 \\
(08)\end{array}$ & $\begin{array}{c}2,52 \\
(06)\end{array}$ & $\begin{array}{l}1,05 \\
(06)\end{array}$ & $\begin{array}{l}0,66 \\
(09)\end{array}$ & $\begin{array}{c}0,99 \\
(06)\end{array}$ & $\begin{array}{l}1,28 \\
(07)\end{array}$ & $\begin{array}{l}1,33 \\
(08)\end{array}$ & $\begin{array}{c}2,91 \\
(15)\end{array}$ & $\begin{array}{l}3,38 \\
(08)\end{array}$ & $\begin{array}{l}0,86 \\
(02)\end{array}$ & $\begin{array}{l}1,88 \\
\text { (11) }\end{array}$ & $\begin{array}{l}1,25 \\
(06)\end{array}$ & $\begin{array}{l}0,41 \\
(03)\end{array}$ & $\begin{array}{l}0,00 \\
(0)\end{array}$ & $\begin{array}{c}0,00 \\
(0)\end{array}$ \\
\hline & Tarde & $\begin{array}{l}1,18 \\
(12)\end{array}$ & $\begin{array}{l}0,84 \\
(02)\end{array}$ & $\begin{array}{l}1,40 \\
(08)\end{array}$ & $\begin{array}{l}0,37 \\
(05)\end{array}$ & $\begin{array}{l}0,66 \\
(04)\end{array}$ & $\begin{array}{l}1,10 \\
(06)\end{array}$ & $\begin{array}{l}0,67 \\
(04)\end{array}$ & $\begin{array}{c}0,00 \\
(0)\end{array}$ & $\begin{array}{c}0,42 \\
(01)\end{array}$ & $\begin{array}{l}0,43 \\
(01)\end{array}$ & $\begin{array}{l}0,51 \\
(03)\end{array}$ & $\begin{array}{c}0,00 \\
(0)\end{array}$ & $\begin{array}{c}0,00 \\
(0)\end{array}$ & $\begin{array}{l}0,19 \\
(01)\end{array}$ & $\begin{array}{c}0,00 \\
(0)\end{array}$ \\
\hline
\end{tabular}

Fonte: os autores (2021)

A existência de correlações (Pearson) entre a taxa geral de uso diurno e as taxas de conexão visual, conexão funcional, supervisão por sacadas e de barreira física e visual, considerando as 15 quadras (Tabela 01), indicam que quanto maior a extensão de janelas e portas de vidro que permitem a visualização do espaço aberto público a partir do interior das edificações (conexão visual), a quantidade de acessos de pedestres às edificações (conexão funcional), e de sacadas (que permitem a supervisão das ruas), maior a presença de pessoas nas quadras; por outro lado, quanto maior a extensão linear de muros e paredes cegas (barreira físicas), menor a presença de pessoas.

Isso explica os diferentes padrões encontrados nas quadras tipo 1 e nas tipo 3. As quadras 1D, 1E e 1F, possuem taxas de barreiras físicas e visuais maiores que aquelas nas quadras $1 \mathrm{~A}, 1 \mathrm{~B}$ e $1 \mathrm{C}$, que por sua vez possuem maiores taxas de conexão visual e funcional (Figura 1). Por sua vez, enquanto o lado delimitado por condomínio fechado na quadra $3 \mathrm{~A}$ possui acesso ao empreendimento, o lado com este tipo de condomínio na quadra 3B não possui acesso (Figura 1). 
Tabela 1 - Correlações Pearson

\begin{tabular}{|c|c|c|c|c|c|c|c|c|}
\hline & $\begin{array}{l}\text { TAXAS } \\
\text { DE USO }\end{array}$ & Geral & $\begin{array}{l}\text { Semana } \\
\text { Manhã }\end{array}$ & $\begin{array}{c}\text { Semana } \\
\text { Tarde }\end{array}$ & $\begin{array}{l}\text { Sábados } \\
\text { Manhã }\end{array}$ & $\begin{array}{l}\text { Sábados } \\
\text { Tarde }\end{array}$ & $\begin{array}{l}\text { Domingo } \\
\text { Manhã }\end{array}$ & $\begin{array}{c}\text { Domingo } \\
\text { Tarde }\end{array}$ \\
\hline \multirow{2}{*}{$\begin{array}{c}\text { TAXA } \\
\text { Conexão } \\
\text { Visual }\end{array}$} & $\begin{array}{l}\text { Coef. } \\
\text { Pearson }\end{array}$ & 0,772 & 0,702 & 0,705 & \multirow{2}{*}{ n.sig } & 0,557 & \multirow{2}{*}{ n.sig } & 0,595 \\
\hline & Sig & 0,001 & 0,003 & 0,003 & & 0,031 & & 0,019 \\
\hline \multirow{2}{*}{$\begin{array}{c}\text { TAXA } \\
\text { Conexão } \\
\text { Funcional }\end{array}$} & $\begin{array}{l}\text { Coef. } \\
\text { Pearson }\end{array}$ & 0,809 & 0,708 & 0,731 & \multirow{2}{*}{ n.sig } & 0,528 & \multirow{2}{*}{ n.sig } & 0,584 \\
\hline & Sig & 0,000 & 0,003 & 0,002 & & 0,043 & & 0,022 \\
\hline \multirow{2}{*}{$\begin{array}{c}\text { TAXA } \\
\text { Supervisão } \\
\text { sacada }\end{array}$} & $\begin{array}{l}\text { Coef. } \\
\text { Pearson }\end{array}$ & 0,568 & 0,777 & 0,645 & \multirow{2}{*}{ n.sig } & 0,653 & 0,606 & ncio \\
\hline & Sig & 0,027 & 0,001 & 0,009 & & 0,008 & 0,017 & \\
\hline \multirow{2}{*}{$\begin{array}{c}\text { TAXA } \\
\text { Barreira Física }\end{array}$} & $\begin{array}{l}\text { Coef. } \\
\text { Pearson }\end{array}$ & $-0,722$ & $-0,710$ & $-0,790$ & \multirow{2}{*}{ n.sig } & $-0,575$ & \multirow{2}{*}{ n.sig } & $-0,653$ \\
\hline & Sig & 0,002 & 0,003 & 0,000 & & 0,025 & & 0,008 \\
\hline
\end{tabular}

Fonte: os autores (2021)

Os resultados dos dias da semana pela manhã seguem a mesma tendência do uso geral diurno. Mesmo que a taxa da quadra $2 \mathrm{~B}$, seja claramente mais elevada que a da quadra $2 \mathrm{~A}$, as duas continuam entre as mais elevadas. Ainda, as quadras tipo 1, assim como as tipo 3 e as tipo 4, apresentam os mesmos padrões anteriores (Gráfico 01). Já, quanto aos dias da semana pela tarde, as taxas mais elevadas são claramente as das quadras $1 \mathrm{~A}$ e $1 \mathrm{C}$, até quase o dobro das segundas mais elevadas, aquelas nas quadras $1 \mathrm{~B}, 1 \mathrm{~F}, 2 \mathrm{~B}$ e $3 \mathrm{~A}$, logo seguidas por aquelas nas quadras 1D, 1E, e 3B. Todavia, tanto em relação ao turno da manhã quanto ao da tarde, as duas quadras do tipo 4 e as três do tipo 5 continuam sendo as que possuem as taxas de uso mais baixas. Também foram encontradas correlações (Pearson) entre as taxas de uso dos dias da semana, durante os períodos da manhã e tarde (Tabela 1) e as taxas de conexão visual, conexão funcional, supervisão por sacadas e barreira física.

A alteração mais significativa dos finais de semana, tanto pelo período da manhã quanto da tarde, em relação aos dias de semana, é que as duas quadras tipo 4 possuem taxas mais elevadas se comparadas com as dos dias da semana, principalmente a $4 \mathrm{~A}$, o que é explicado pelo maior uso da praça (Figura 1), equipamento que atrai atividades esportivas e de lazer nesses dias, o que também explica as altas taxas das quadras tipo 2 (principalmente aos sábados). Por outro lado, as taxas mais baixas continuam sendo as das três quadras tipo 5 (5A, 5B e 5C), a maioria nula. Adicionalmente, foram encontradas algumas correlações (Pearson) entre as taxas de uso dos sábados e domingos (Tabela1), e as taxas de conexão visual, conexão funcional, supervisão por sacadas e barreira física.

\section{CONCLUSÕES}

Os resultados apresentados evidenciam o impacto negativo dos condomínios fechados murados sobre o uso dos espaços abertos públicos, aferido pela baixa intensidade de uso, quase nula, das quadras caracterizadas em ambos os lados por barreiras físicas e visuais contínuas. Ainda, os resultados reforçam a importância da permeabilidade física e visual, além das sacadas das edificações, para um maior uso dos 
espaços abertos públicos de áreas residenciais, uma vez que a intensidade de uso das quadras investigadas aumenta conforme aumenta o número e/ou a extensão desses elementos, e diminui conforme aumenta a extensão das barreiras físicas e visuais. Assim, este estudo contribui para reforçar a importância das características arquitetônicas das interfaces das edificações para a vitalidade urbana, e, logo, da inadequação da continuação da construção de condomínios delimitados por muros em áreas urbanas consolidadas.

\section{REFERÊNCIAS}

ANTOCHEVIZ, F. B.; FIGUEIREDO, C. Á. de; REIS, A. T. Transformações de interfaces térreas, uso e percepção de segurança em cidade litorânea. Urbe. Revista Brasileira de Gestão urbana, [s. 1.], v. 11, n. e20170225, p. 1-23, 2019. Disponível em: https://doi.org/10.1590/2175-3369.011.001.AO14

BECKER, D. Condomínios Horizontais Fechados: Avaliação de Desempenho Interno e Impacto Físico Espacial no Espaço Urbano. Porto Alegre: Dissertação (Mestrado em Planejamento Urbano e Regional) Programa de Pós-graduação em Planejamento Urbano e Regional (PROPUR), Faculdade de Arquitetura e Urbanismo, Universidade Federal do Rio Grande do Sul, 2005.

BENTLEY, I.; et al. Responsive environments. London: The Architectural Press Ltda., 1985.

BORSDORF, A. Cómo modelar el desarrolo y la dinámica de la cuidad latinoamericana. Eure, [s. 1.], v. 29, n. 86, 2003. Disponível em: https://doi.org/10.4067/s0250-71612003008600002

GEHL, J. Cities for people. Whashington: Island Press, 2010.

GEHL, J. Life Between Buildings: Using Public Space. New York: Van Nostrand Reinhold Company, 1987. ISSN 0277-2426.v. 8 Disponível em: https://doi.org/10.3368/lj.8.1.54

GEHL, J.; KAEFER, L. J.; REIGSTAD, S. Close encounters with buildings. Urban Design International, [s. 1.], v. 11, n. 1, p. 29-47, 2006. Disponível em: https://doi.org/10.1057/palgrave.udi.9000162

GRANT, J.; MITTELSTEADT, L. Types of gated communities. Environment and Planning B: Planning and Design, [s. 1.], v. 31, n. 6, p. 913-930, 2004. Disponível em: https://doi.org/10.1068/b3165

HILLIER, B.; HANSON, J. The social logic of space. London: Cambridge University Press, 1984. Disponível em: https://doi.org/10.1017/cbo9780511597237

HERTZBERG, Herman. Lições de Arquitetura. São Paulo: Martins Fontes, 1999.

JACOBS, Jane. Morte e Vida de Grandes Cidades. São Paulo: Martins Fontes, 2000.

$\mathrm{KOCH}, \mathrm{M}$. R. Condomínios Fechados: as novas configurações do urbano e a dinâmica imobiliária. Indicadores Econômicos FEE, [s. 1.], v. 35, n. 3, p. 99-115, 2008.

M. ESTEVES, A. V.; NOGUEIRA, M. A proliferação e a consolidação de condomínios fechados: um estudo de caso em uma cidade média - Divinópolis ( MG ). Geografias, [s. 1.], v. 9, p. 23-39, 2013. 
MAMMARELLA, R.; BARCELOS, T. M. Padrões sociais de territorialidade e condomínios fechados na Metrópole Gaúcha. Textos para Discussão FEE, [s. 1.], n. 46, p. 189-194, 2008.

REIS, A. T.; BECKER, D. Morfologia urbana e o impacto dos condomínios fechados. Revista Projectare, [s. 1.], p. 108-119, 2011.

UEDA, V. Os novos empreendimentos imobiliários e as transformações recentes no espaço urbano de Porto Alegre. In: , 2005, São Paulo. X Encontro de Geógrafos da América Latina. São Paulo: [s. n.], 2005. p. 1594815965. Disponível em: https://doi.org/10.1344/sn2005.9.929

WEBSTER, C.; GLASZE, G.; FRANTZ, K. Guest editorial. Environment and Planning B: Planning and Design, [s. 1.], v. 29, n. 3, p. 315-320, 2002. Disponível em: https://doi.org/10.1068/b12926 